\title{
A Regime Switching Model for the Term Structure of Credit Risk Spreads
}

\author{
Seungmook Choi ${ }^{1}$, Michael D. Marcozzi² \\ ${ }^{1}$ Department of Finance, University of Nevada Las Vegas, Las Vegas, NV, USA \\ ${ }^{2}$ Department of Mathematical Sciences, University of Nevada Las Vegas, Las Vegas, NV, USA \\ Email: seungmook.choi@unlv.edu, marcozzi@unlv.nevada.edu
}

Received 22 January 2015; accepted 10 February 2015; published 13 February 2015

Copyright (C) 2015 by authors and Scientific Research Publishing Inc.

This work is licensed under the Creative Commons Attribution International License (CC BY). http://creativecommons.org/licenses/by/4.0/

(c) (i) Open Access

\begin{abstract}
We consider a rating-based model for the term structure of credit risk spreads wherein the creditworthiness of the issuer is represented as a finite-state continuous time Markov process. This approach entails a progressive drift in credit quality towards default. A model of the economy is presented featuring stochastic transition probabilities; credit instruments are valued via an ultra parabolic Hamilton-Jacobi system of equations discretized utilizing the method-of-lines finite difference method. Computations for a callable bond are presented demonstrating the efficiency of the method.
\end{abstract}

Keywords

Optimal Stopping, Failure Rate, Regime Switching, Credit Risk Spreads

\section{Introduction}

When pricing of credit instruments subject to default risk, market participants typically assume that default is unpredictable, using dynamics derived from rating information in order to take advantage of credit events (cf. [1]). Generally, they fall into a loose hierarchy known as reduced-form models. The most ubiquitous approach involving hazard rate models wherein default risk via unexpected events is modeled by a jump process. In this framework, credit-risky securities are priced as discounted expectation under the risk neutral probability measure with modified discount rate (cf. [2], [3]). Although conceptually simple and easy to implement, these models are limited by the appropriate calibration of the hazard rate process. More generally, spread modeling represents spreads directly and eliminates the need to make assumptions on recovery (cf. [4], [5]). Finally, rating based models consider the creditworthiness of the issuer to be a key state variable used to calibrate the risk-neutral hazard rate (cf. [6]-[8]). A progressive drift in credit quality toward default (an absorbing state) is 
now allowed as opposed to a single jump to bankruptcy, as in many hazard rate models. Rating based models are particularly useful for the pricing of securities whose payoffs depend on the rating of the issuer.

In this paper, we consider a rating based regime switching model for the term-structure of credit risk spreads in continuous time (cf. [9], [10]). A unique feature of our model is the inclusion of stochastic transition probabilities. Credit instruments are then characterized as the solution to a ultraparabolic Hamilton-Jacobi system of equations for which we develop a methods-of-lines finite difference method. Computations are presented for a rating based callable bond which validates the applicability and efficiency of the method.

\section{Model of the Economy}

In this section, we introduce the dynamics of the risk-less and risky term structures of interest rates as well as the bankruptcy process. To this end, we assume the existence of a unique equivalent martingale measure such that all risk-less and risky zero-coupon bond prices are martingales after normalization by the money market account (cf. [11], [12]). Without loss of generality, we suppose a single risky zero-coupon bond price and continuous trading over a finite time interval $[0, \tilde{T}]$. We let $\mathcal{E}(t) \quad(0<t<\tilde{T})$ denote a continuous time Markov process on the regime (or états) space $\mathbb{I}_{m}=\{0,1,2, \cdots, m\}$ with associated transition probabilities

$P_{i j}(t)=\operatorname{Pr}\{\mathcal{E}(t+\Delta t)=j \mid \mathcal{E}(t)=i\}$, for all $\Delta t>0$; it follows that

$$
0 \leq P_{i j} \quad \text { and } \quad \sum_{j=0}^{m} P_{i j}=1,
$$

for $i \in \mathbb{I}_{m}$. Let $\boldsymbol{P}_{i}(t)=\left(P_{i 0}, P_{i 1}, \cdots, P_{i m}\right)$ represent the $i^{\text {th }}$-state transition distribution.

We define the transition probabilities as follows. The $0^{\text {th }}$-state we associate with default, in which case $\boldsymbol{P}_{0}(s)=(1,0, \cdots, 0)$. For $i=1,2, \cdots, m$, we define the $i^{\text {th }}$-state transition dynamics consistent with the nonnegativity constraint in (2.1) such that $(j=1,2, \cdots, m-1)$

$$
\begin{aligned}
& \mathrm{d} P_{i j}(s)=\alpha_{i j}\left(\bar{p}_{i j}-P_{i j}\right) \mathrm{d} s+\sigma_{i j} \beta_{j}\left(\boldsymbol{P}_{i}\right) \mathrm{d} W_{i j}(s), \\
& P_{i j}(t)=p_{i j} \in(0,1),
\end{aligned}
$$

for $0<t<s<\tilde{T}$, where

$$
\beta_{j}^{2}\left(\boldsymbol{P}_{i}\right)= \begin{cases}P_{i j} & \text { if } j<m-1 \\ P_{i j}\left(1-\sum_{\varepsilon=1}^{m-1} P_{i \varepsilon}\right) & \text { if } j=m-1\end{cases}
$$

and $0<\bar{p}_{i j}$ is the mean transition level satisfying $\sum_{\varepsilon=1}^{m-1} \bar{p}_{i \varepsilon} \leq 1,0 \leq \alpha_{i j}$ is the rate of reversion to the mean, $0 \leq \sigma_{i j}$ and $\mathrm{d} W_{i j}$ is a Wiener process. From (2.1), it follows that $P_{i m}=1-P_{i 0}-P_{i 1}-\cdots-P_{i m-1}$ and so

$$
\begin{aligned}
& \mathrm{d} P_{i m}(s)=-\sum_{j=1}^{m-1} \alpha_{i j}\left(\bar{p}_{i j}-P_{i j}\right) \mathrm{d} s-\sum_{j=1}^{m-1} \sigma_{i j} \beta_{j}\left(\boldsymbol{P}_{i}\right) \mathrm{d} W_{i j}(s), \\
& P_{i m}(t)=p_{i m}=1-\sum_{j=1}^{m-1} p_{i j} \in(0,1) .
\end{aligned}
$$

We relate the transition matrix $\Pi=\left(P_{i j}\right)$ to the regime dynamics via the infinitesimal generator $\Lambda$,

$$
\Lambda=\lim _{h \rightarrow 0^{+}} \frac{\Pi(h)-\boldsymbol{I}}{h},
$$

such that

$$
\frac{\mathrm{d} \boldsymbol{P}(s)}{\mathrm{d} s}=\boldsymbol{P}(s) \Lambda,
$$

for $0<t<s<\tilde{T}$, and

$$
\boldsymbol{P}(t)=\boldsymbol{P},
$$


where $\boldsymbol{P}=\left(P_{0}, P_{1}, \cdots, P_{m}\right)$ is the vector of probabilities $P_{j}(s)=\operatorname{Pr}\{E(s)=j\}$. Without loss of generality, we associate $E(s)$ with the vector $\mathcal{E}(s) \in\left\{\boldsymbol{e}_{0}, \boldsymbol{e}_{1}, \cdots, \boldsymbol{e}_{m}\right\}, \boldsymbol{e}_{0}=(1,0, \cdots, 0), \boldsymbol{e}_{1}=(0,1, \cdots, 0), \cdots$, $\boldsymbol{e}_{m}=(0,0, \cdots, 1)$, subject to the dynamics

$$
\begin{aligned}
& \mathrm{d} \mathcal{E}(s)=\mathcal{E}(s) \Lambda \mathrm{d} s+\mathrm{d} \boldsymbol{M}(s), \\
& \mathcal{E}(t)=\boldsymbol{e},
\end{aligned}
$$

for $0<t<s<\tilde{T}$, where $\boldsymbol{M}(s)$ is a martingale with respect to the filtration generated by $\mathcal{E}$ and $\boldsymbol{P}(s)=\mathbb{E}[\mathcal{E}(s)]$ ([13], Chap 4.8; [14], Part III, App. B; [15], Chap 8). In particular, the state of the system is known at inception such that $\boldsymbol{e}_{i}=\mathbb{E}[\mathcal{E}(t)]=\boldsymbol{P}(t)=\boldsymbol{P}$, for some $i \in \mathbb{I}_{m}$.

We suppose that the risky interest rate $R$ follows a state specific Cox-Ingersall-Ross dynamic given by

$$
\mathrm{d} R(s ; \mathcal{E})=\alpha(\mathcal{E}) \cdot(\bar{r}(\mathcal{E})-R) \mathrm{d} s+\sigma(\mathcal{E}) \sqrt{R} \cdot \mathrm{d} W(s)
$$

for $0<t<s<\tilde{T}$, with mean reversion level $\bar{r}(\mathcal{E})$ and rate of reversion to the mean $\alpha(\mathcal{E})$, such that

$$
R(t ; \boldsymbol{e})=r(\boldsymbol{e}),
$$

where $\mathrm{d} W$ is a Wiener process. In default $\alpha\left(\boldsymbol{e}_{0}\right)=\sigma\left(\boldsymbol{e}_{0}\right)=0$, otherwise $\alpha\left(\boldsymbol{e}_{i}\right)=\alpha_{i}$ and $\sigma\left(\boldsymbol{e}_{i}\right)=\sigma_{i}$. The risky bond price $B$ associated with a maturity $T$ satisfies

$$
\begin{aligned}
\frac{\mathrm{d} B(s)}{\mathrm{d} s} & =R(s ; \boldsymbol{e}) B(s), \\
B(t) & =b .
\end{aligned}
$$

We consider the risk-less interest rate $\varrho$ to satisfy

$$
\begin{aligned}
& \mathrm{d} \varrho(s ; \mathcal{E}) \mathrm{d} s=0, \\
& \varrho(t ; \mathcal{E})=\rho(\mathcal{E}),
\end{aligned}
$$

where in default $\rho\left(\boldsymbol{e}_{0}\right)=0$ for convenience, and $\rho\left(\boldsymbol{e}_{i}\right)=\rho$ otherwise.

For a given contract $\psi$, we define the value function associated with the joint Markov ultradiffusion process (2.2)-(2.5) such that

$$
v\left(t, b, r, \boldsymbol{\pi}, \boldsymbol{e}_{i}\right)=\mathbb{E}\{\exp [-\varrho(\mathcal{E}) \cdot(T-t)] \cdot \psi(T, B(T), R(T), \Pi(T), \mathcal{E})\},
$$

for $0<t<T<\tilde{T}$, where $\pi=\left(p_{i j}\right)$.

In particular, for a non-coupon paying bond $\psi\left(T, \boldsymbol{e}_{0}\right)=\delta$ and $\psi\left(T, \boldsymbol{e}_{i}\right)=1$ otherwise, where $\delta$ is the default recovery rate, whereas for a callable bond $\psi\left(T, b, \boldsymbol{e}_{0}\right)=0$ and $\psi\left(T, b, \boldsymbol{e}_{i}\right)=\max \left\{b-E\left(\boldsymbol{e}_{i}\right), 0\right\}$ otherwise, for some rating based exercise price $E\left(\boldsymbol{e}_{i}\right)$. Generalization of (2.6) and the subsequent analysis to include early exercise features follows routinely and will not be considered here.

\section{Characterization}

Letting $v_{i}(t, b, r, \boldsymbol{\pi})=v\left(t, b, r, \boldsymbol{\pi}, \boldsymbol{e}_{i}\right)$ and

$$
\boldsymbol{v}(t, \cdot)=\left(v_{0}(t, \cdot), v_{1}(t, \cdot), \cdots, v_{m}(t, \cdot)\right)^{\mathrm{T}},
$$

we recover (2.6) succinctly as

$$
v\left(t, \cdot, \boldsymbol{e}_{i}\right)=\boldsymbol{e}_{i} \cdot \boldsymbol{v}(t, \cdot),
$$

for $i \in \mathbb{I}_{m}$. By Itô's rule, the value function (2.6) is characterized via (3.1) as the solution to the ultraparabolic Hamilton-Jacobi system of equations

$$
\begin{aligned}
& \frac{\partial v_{0}}{\partial t}=0 \\
& v_{0}(T, x)=\psi_{0}(T, x)
\end{aligned}
$$




$$
\begin{aligned}
& \frac{\partial v_{1}}{\partial t}+r b \frac{\partial v_{1}}{\partial b}+\mathcal{A}_{1} v_{1}+p_{10} v_{0}+\left(p_{11}-1\right) v_{1}+\cdots+p_{1 m} v_{m}=0 \\
& v_{1}(T, x)=\psi_{1}(T, x) \\
& \vdots \\
& \frac{\partial v_{m}}{\partial t}+r b \frac{\partial v_{m}}{\partial b}+\mathcal{A}_{m} v_{m}+p_{m 0} v_{0}+p_{m 1} v_{1}+\cdots+\left(p_{m m}-1\right) v_{m}=0 \\
& v_{m}(T, x)=\psi_{m}(T, x),
\end{aligned}
$$

where

$$
\begin{aligned}
\mathcal{A}_{i} v= & \frac{1}{2} r \sigma_{i}^{2} \frac{\partial^{2} v}{\partial r^{2}}+\alpha_{i}\left(\bar{r}_{i}-r\right) \frac{\partial v}{\partial r}+\sum_{1 \leq j<m} \frac{1}{2} \beta_{j}^{2}\left(\boldsymbol{P}_{i}\right) \sigma_{i j}^{2} \frac{\partial^{2} v}{\partial p_{i j}^{2}} \\
& +\sum_{1 \leq j<m} \alpha_{i j}\left(\bar{p}_{i j}-p_{i j}\right) \frac{\partial v}{\partial p_{i j}}-\sum_{1 \leq j<m} \frac{1}{2} \beta_{j}^{2}\left(\boldsymbol{P}_{i}\right) \sigma_{i j}^{2} \frac{\partial^{2} v}{\partial p_{i m}^{2}} \\
& -\sum_{1 \leq j<m} \alpha_{i j}\left(\bar{p}_{i j}-p_{i j}\right) \frac{\partial v}{\partial p_{i m}}-\rho\left(\boldsymbol{e}_{\varepsilon}\right) v .
\end{aligned}
$$

Let $\boldsymbol{t}=(t, b) \in \mathcal{Q}=(0, T) \times(0, \infty)$ denote the temporal variable and $\boldsymbol{x}=\left(r, p_{11}, p_{12}, \cdots, p_{m m}\right) \in \Omega=(0, \infty) \times\left\{(0,1)^{m} \mid \sum_{j} p_{1 j} \leq 1\right\} \times \cdots \times\left\{(0,1)^{m} \mid \sum_{j} p_{m j} \leq 1\right\}$ the spatial, we define

$$
\mathcal{H}=\frac{\partial}{\partial t}+r b \frac{\partial}{\partial b}
$$

and $\mathcal{A}=\left(A_{1}, A_{2}, \cdots, A_{m}\right)$, such that the above can be written

$$
\mathcal{H} v(t, x)+\mathcal{A} \cdot v(t, x)+(\Pi-I) \cdot v(t, x)=\mathbf{0},
$$

for all $(\boldsymbol{t}, \boldsymbol{x}) \in \mathcal{Q} \times \Omega$, subject to the terminal constraint

$$
\boldsymbol{v}(T, \cdot)=\psi(T, \cdot),
$$

for $(b, \boldsymbol{x}) \in(0, \infty) \times \Omega$, where $\psi=\left(\psi\left(\cdot, \boldsymbol{e}_{0}\right), \psi\left(\cdot, \boldsymbol{e}_{1}\right), \cdots, \psi\left(\cdot, \boldsymbol{e}_{m}\right)\right)^{\mathrm{T}}$.

\section{Approximation Solvability}

Towards obtaining a constructive approximation of (3.2), we consider an exhaustive sequence of bounded open domains $\left\{\Omega_{k}\right\}$ such that $\Omega_{k} \subset \Omega_{k+1}$ and $\cup \Omega_{k}=\Omega$ as well as a sequence of monotonically increasing real numbers $T_{k} \rightarrow \infty$, as $k \rightarrow \infty$. Let $\mathcal{Q}_{k}=(0, T) \times\left(0, T_{k}\right)$ and $\partial \mathcal{Q}_{k}=\{T\} \times\left(0, T_{k}\right) \cup(0, T) \times\left\{T_{k}\right\}$, we seek $\boldsymbol{v}_{k}(\boldsymbol{t}, \boldsymbol{x})$ satisfying

$$
\mathcal{H} \boldsymbol{v}_{k}(\boldsymbol{t}, \boldsymbol{x})+\mathcal{A} \cdot \boldsymbol{v}_{k}(\boldsymbol{t}, \boldsymbol{x})+(\Pi-\boldsymbol{I}) \cdot \boldsymbol{v}_{k}(\boldsymbol{t}, \boldsymbol{x})=\mathbf{0},
$$

for all $(\boldsymbol{t}, \boldsymbol{x}) \in \mathcal{Q}_{k} \times \Omega_{k}$, subject to the boundary condition

$$
v_{k}(t, x)=\psi(t, x)
$$

for $(\boldsymbol{t}, \boldsymbol{x}) \in \mathcal{Q}_{k} \times \partial \Omega_{k}$, and terminal constraint

$$
v_{k}(T, x)=\psi(T, x),
$$

where $(\boldsymbol{T}, \boldsymbol{x}) \in \partial \mathcal{Q}_{k} \times \Omega_{k}$. As (3.2) is an infinite horizon problem in $b$, we remark to the necessity of introducing the artificial terminal condition $\boldsymbol{v}_{k}=\psi$ along the frontier $(T, b) \in\{T\} \times\left(0, T_{k}\right)$ (cf. [16]). In particular, $\boldsymbol{v}_{k}(\boldsymbol{t}, \boldsymbol{x}) \rightarrow \boldsymbol{v}(\boldsymbol{t}, \boldsymbol{x})$ as $k \rightarrow \infty$, on any compact subset of $\Omega$, for any fixed $\boldsymbol{t} \in \overline{\mathcal{Q}}$.

We next place (4.1) into standard form by setting $\tau=T-t, \varsigma_{k}=T_{k}-b, \tau=\left(\tau, \varsigma_{k}\right)$, in which case $\boldsymbol{u}_{k}\left(T-\tau, T_{k}-b, \cdot\right)=\boldsymbol{v}_{k}(t, b, \cdot)$. Letting 


$$
\mathcal{H}_{k}=\frac{\partial}{\partial \tau}+r\left(T_{k}-\varsigma_{k}\right) \frac{\partial}{\partial \varsigma_{k}}
$$

Equation (4.1) becomes

$$
-\mathcal{H}_{k} \boldsymbol{u}_{k}(\tau, \boldsymbol{x})+\mathcal{A} \cdot \boldsymbol{u}_{k}(\tau, \boldsymbol{x})+(\Pi-\boldsymbol{I}) \boldsymbol{u}_{k}(\tau, \boldsymbol{x})=\mathbf{0},
$$

for all $(\tau, x) \in \mathcal{Q}_{k} \times \Omega_{k}$, subject to the boundary condition

$$
\mathbf{u}_{k}(\tau, x)=\psi(\tau, x)
$$

for $(\tau, \boldsymbol{x}) \in \mathcal{Q}_{k} \times \partial \Omega_{k}$, and initial condition

$$
\mathbf{u}_{k}\left(\tau_{0}, x\right)=\psi\left(\tau_{0}, x\right)
$$

where $\left(\tau_{0}, \boldsymbol{x}\right) \in \partial \mathcal{Q}_{0, k} \times \Omega_{k}$, where $\partial \mathcal{Q}_{0, k}=\{0\} \times\left(0, T_{k}\right) \cup(0, T) \times\{0\}$.

We consider the discretization of (4.2) by the backward Euler method temporally and central differencing in space. To this end, we introduce the temporal step sizes $\left(\delta_{\tau}, \delta_{\zeta}\right) \in \mathbb{R}_{+}^{2}$ and mesh sizes $\left(\mathcal{N}_{\tau}, \mathcal{N}_{\zeta}\right) \in \mathbb{N}^{2}$, such that $T=\delta_{\tau} \cdot \mathcal{N}_{\tau}$ and $T_{k}=\delta_{\varsigma} \cdot \mathcal{N}_{\varsigma}$. Spatially, we utilize the step sizes $\left(\delta_{r}, \delta_{p}\right) \in \mathbb{R}_{+}^{2}$ and mesh sizes $\left(\mathcal{M}_{r}, \mathcal{M}_{p}\right) \in \mathbb{N}^{2}$; we denote the value of $\boldsymbol{u}_{k}$ on the grid by

$$
\boldsymbol{u}_{k}^{\nu_{1}, \nu_{2}, \mu_{0}, \mu_{1}, \cdots, \mu_{m}^{2}}=\boldsymbol{u}_{k}\left(\tau^{\nu_{1}}, \varsigma^{\nu_{2}}, r^{\mu_{0}}, p_{11}^{\mu_{1}}, \cdots, p_{m m}^{\mu_{m} m^{2}}\right),
$$

where $\tau^{v_{1}}=v_{1} \cdot \delta_{\tau}, \varsigma^{\nu_{2}}=v_{2} \cdot \delta_{\zeta}, r^{\mu_{0}}=\mu_{0} \cdot \delta_{r}, \quad p_{11}^{\mu_{1}}=\mu_{1} \cdot \delta_{p}$, and so forth. Notationally, we let $(\boldsymbol{v}, \boldsymbol{\mu}) \in \mathcal{Q}_{\delta} \times \Omega_{k, \delta}$, where $\boldsymbol{v}=\left(v_{1}, v_{2}\right), \quad \boldsymbol{\mu}=\left(\mu_{0}, \mu_{1}, \cdots, \mu_{m^{2}}\right), \quad \mathcal{Q}_{\delta}=\left[0,1, \cdots, \mathcal{N}_{\tau}\right] \times\left[0,1, \cdots, \mathcal{N}_{\varsigma}\right]$, and $\Omega_{k, \delta}=\left[0,1, \cdots, \mathcal{M}_{r}\right] \times\left\{\left[0,1, \cdots, \mathcal{M}_{p}\right]^{m} \mid \sum_{j} p_{1 j}^{\mu_{1}} \leq 1\right\}^{m} \times \cdots \times\left\{\left[0,1, \cdots, \mathcal{M}_{p}\right]^{m} \mid \sum_{j} p_{m j}^{\mu_{m}} \leq 1\right\}^{m}$. For

$$
\boldsymbol{u}_{k}(\boldsymbol{v}, \boldsymbol{\mu})=\boldsymbol{u}_{k}^{v_{1}, v_{2}, \mu_{0}, \mu_{1}, \cdots, \mu_{m^{2}}},
$$

the difference quotients are then backward first order in time:

$$
\begin{aligned}
& \nabla_{\tau} \boldsymbol{u}_{k}^{v_{1}, v_{2}, \mu_{0}, \mu_{1}, \cdots, \mu_{m}{ }^{2}}=\frac{1}{\delta_{\tau}}\left[\boldsymbol{u}_{k}^{v_{1}, \nu_{2}, \mu_{0}, \mu_{1}, \cdots, \mu_{m^{2}}}-\boldsymbol{u}_{k}^{v_{1}-1, v_{2}, \mu_{0}, \mu_{1}, \cdots, \mu_{m^{2}}}\right] \\
& \nabla_{\varsigma} \boldsymbol{\mu}_{k}^{v_{1}, v_{2}, \mu_{0}, \mu_{1}, \cdots, \mu_{m^{2}}}=\frac{1}{\delta_{\tau}}\left[\boldsymbol{\mu}_{k}^{v_{1}, \nu_{2}, \mu_{0}, \mu_{1}, \cdots, \mu_{m^{2}}}-\boldsymbol{\mu}_{k}^{v_{1}, \nu_{2}-1, \mu_{0}, \mu_{1}, \cdots, \mu_{m^{2}}}\right]
\end{aligned}
$$

and central second-order in space:

$$
\begin{aligned}
& \delta_{0}^{2} \boldsymbol{\mu}_{k}^{\nu_{1}, \nu_{2}, \mu_{0}, \mu_{1}, \cdots, \mu_{m^{2}}}=\frac{1}{\delta_{r}^{2}}\left[\boldsymbol{\mu}_{k}^{\nu_{1}, \nu_{2}, \mu_{0}+1, \mu_{1}, \cdots, \mu_{m^{2}}}-\boldsymbol{\mu}_{k}^{\nu_{1}, \nu_{2}, \mu_{0}, \mu_{1}, \cdots, \mu_{m^{2}}}+\boldsymbol{\mu}_{k}^{v_{1}, \nu_{2}, \mu_{0}-1, \mu_{1}, \cdots, \mu_{m^{2}}}\right] \\
& \delta_{1}^{2} \boldsymbol{\mu}_{k}^{\nu_{1}, \nu_{2}, \mu_{0}, \mu_{1}, \cdots, \mu_{m^{2}}}=\frac{1}{\delta_{r}^{2}}\left[\boldsymbol{\mu}_{k}^{\nu_{1}, \nu_{2}, \mu_{0}, \mu_{1}+1, \cdots, \mu_{m^{2}}}-\boldsymbol{\mu}_{k}^{\nu_{1}, \nu_{2}, \mu_{0}, \mu_{1}, \cdots, \mu_{m^{2}}}+\boldsymbol{\mu}_{k}^{\nu_{1}, \nu_{2}, \mu_{0}, \mu_{1}-1, \cdots, \mu_{m^{2}}}\right]
\end{aligned}
$$

and so forth, and

$$
\begin{aligned}
& \delta_{0} \boldsymbol{\mu}_{k}^{v_{1}, \nu_{2}, \mu_{0}, \mu_{1}, \cdots, \mu_{m^{2}}}=\frac{1}{2 \delta_{r}}\left[\boldsymbol{\mu}_{k}^{v_{1}, \nu_{2}, \mu_{0}+1, \mu_{1}, \cdots, \mu_{m^{2}}}-\boldsymbol{\mu}_{k}^{v_{1}, v_{2}, \mu_{0}-1, \mu_{1}, \cdots, \mu_{m^{2}}}\right] \\
& \delta_{1} \boldsymbol{\mu}_{k}^{v_{1}, \nu_{2}, \mu_{0}, \mu_{1}, \cdots, \mu_{m^{2}}}=\frac{1}{2 \delta_{r}}\left[\boldsymbol{\mu}_{k}^{\nu_{1}, \nu_{2}, \mu_{0}, \mu_{1}+1, \cdots, \mu_{m^{2}}}-\boldsymbol{\mu}_{k}^{v_{1}, \nu_{2}, \mu_{0}, \mu_{1}-1, \cdots, \mu_{m^{2}}}\right]
\end{aligned}
$$

and so forth.

Given the above, we define the method-of-lines finite difference discretization of (4.2) such that

$$
-\mathcal{H}_{\delta} \boldsymbol{u}_{k}(\boldsymbol{v}, \boldsymbol{\mu})+\mathcal{A}_{\delta} \cdot \boldsymbol{u}_{k}(\boldsymbol{v}, \boldsymbol{\mu})+(\Pi-\boldsymbol{I}) \boldsymbol{u}_{k}(\boldsymbol{v}, \boldsymbol{\mu})=\mathbf{0},
$$

for all $(\boldsymbol{\nu}, \boldsymbol{\mu}) \in \mathcal{Q}_{\delta} \times \Omega_{k, \delta}$, subject to the boundary condition 
for $(\boldsymbol{v}, \boldsymbol{\mu}) \in \mathcal{Q}_{\delta} \times \partial \Omega_{k, \delta}$, and initial condition

$$
u_{k}(v, \mu)=\psi(\tau, x),
$$

$$
\boldsymbol{u}_{k}\left(\boldsymbol{v}_{0}, \mu\right)=\psi\left(\tau_{0}, \boldsymbol{x}\right),
$$

where $(\boldsymbol{v}, \boldsymbol{\mu}) \in \partial \mathcal{Q}_{\delta} \times \Omega_{k, \delta}, \quad \partial \mathcal{Q}_{\delta}=\{0\} \times\left[0,1, \cdots, \mathcal{N}_{\varsigma}\right] \cup\left[0,1, \cdots, \mathcal{N}_{\tau}\right] \times\{0\}$,

$$
\begin{aligned}
\mathcal{H}_{\delta} u=\mathcal{H}_{\delta}(\boldsymbol{v}, \boldsymbol{\mu}) u=\nabla_{\tau} u+r^{\mu_{0}}\left(T_{k}-\varsigma^{\nu_{2}}\right) \nabla_{\varsigma} u, \\
\mathcal{A}_{\varepsilon} u=\mathcal{A}_{\varepsilon}(\boldsymbol{v}, \boldsymbol{\mu}) u=\frac{1}{2} r^{\mu_{0}} \sigma_{\varepsilon}^{2} \delta_{0}^{2} u+\alpha_{\varepsilon}\left(\bar{r}_{\varepsilon}-r^{\mu_{0}}\right) \delta_{0} u+\frac{1}{2} \beta_{1}^{2}\left(\boldsymbol{P}_{1}\right) \sigma_{11}^{2} \delta_{1}^{2} u \\
+\cdots+\frac{1}{2} \beta_{m-1}^{2}\left(\boldsymbol{P}_{m-1}\right) \sigma_{m-1, m-1}^{2} \delta_{(m-1)^{2}}^{2} u-\sum_{1 \leq j<m} \frac{1}{2} \beta_{j}^{2}\left(\boldsymbol{P}_{j}\right) \sigma_{\varepsilon j}^{2} \delta_{m}^{2} u \\
+\alpha_{11}\left(\bar{p}_{11}-p_{11}\right) \delta_{1} u+\cdots+\alpha_{m-1, m-1}\left(\bar{p}_{m-1, m-1}-p_{m-1, m-1}\right) \delta_{(m-1)^{2}} u \\
-\sum_{1 \leq j<m} \alpha_{\varepsilon j}\left(\bar{p}_{\varepsilon j}-p_{\varepsilon j}\right) \delta_{m} u-\rho\left(\boldsymbol{e}_{\varepsilon}\right) u,
\end{aligned}
$$

and $\mathcal{A}_{\delta}=\left(A_{1}, A_{2}, \cdots, A_{m}\right)$. We solve (4.3) utilizing the pseudo-code (cf. [16], [17]):

do $v_{1}=1, \cdots, \mathcal{N}_{\tau}$

do $\eta_{2}=0, \cdots, \mathcal{N}_{\varsigma}$

solve for $\boldsymbol{u}_{k}(\boldsymbol{v}, \boldsymbol{\mu})$ via (4.3).

\section{Numerical Experiment}

In this section, we present a representative computation for the valuation of a callable bond relative to three credit ratings:

and rating's dependent pay-off contract

$$
\mathcal{E}(t)=\left(\begin{array}{l}
e_{0} \\
e_{1} \\
e_{2}
\end{array}\right)=\left(\begin{array}{l}
\text { Default } \\
A \text { rating } \\
B \text { rating }
\end{array}\right)
$$

$$
\psi(T, b, \mathcal{E})= \begin{cases}0 & \text { if } \mathcal{E}=\boldsymbol{e}_{0} \\ \max \{b-0.70,0\} & \text { if } \mathcal{E}=\boldsymbol{e}_{1} \\ \max \{b-0.68,0\} & \text { if } \mathcal{E}=\boldsymbol{e}_{2}\end{cases}
$$

with expiry $T=0.5$. We suppose a solvent risk-free rate of return of $\rho=0.02$. For simplicity, we will consider the following transition matrix

$$
\Pi=\left(\begin{array}{ccc}
1.00 & 0.00 & 0.00 \\
0.0 & 0.95 & 0.05 \\
P_{\text {def }} & 0.10-P_{\text {def }} & 0.9
\end{array}\right)
$$

in which only the default probability $P_{20}=P_{\text {def }}$ is stochastic.

For $0<t<s<T<\tilde{T}$, we have the economy;

$$
\begin{aligned}
& \mathrm{d} P_{\text {def }}(s)=0.01\left(0.05-P_{\text {def }}\right) \mathrm{d} s+0.05 \sqrt{P_{\text {def }}} \sqrt{0.10-P_{\text {def }}} \mathrm{d} W_{\text {def }}(s) ; P_{\text {def }}(t)=p_{\text {def }} \\
& \mathrm{d} R(s ; \mathcal{E})=\alpha(\mathcal{E}) \cdot(\bar{r}(\mathcal{E})-R) \mathrm{d} s+\sigma(\mathcal{E}) \sqrt{R} \cdot \mathrm{d} W(s) ; R(t ; \boldsymbol{e})=r(\boldsymbol{e}), \\
& \mathrm{d} B(s) \mathrm{d} s=R(s) B(s) \mathrm{d} s ; B(t)=b,
\end{aligned}
$$

where

$$
\alpha(\mathcal{E})=\left\{\begin{array}{ll}
0 & \text { if } \mathcal{E}=\boldsymbol{e}_{0} \\
0.010 & \text { if } \mathcal{E}=\boldsymbol{e}_{1} \\
0.005 & \text { if } \mathcal{E}=\boldsymbol{e}_{2}
\end{array} \quad \sigma(\mathcal{E})= \begin{cases}0 & \text { if } \mathcal{E}=\boldsymbol{e}_{0} \\
0.20 & \text { if } \mathcal{E}=\boldsymbol{e}_{1} \\
0.25 & \text { if } \mathcal{E}=\boldsymbol{e}_{2}\end{cases}\right.
$$


and

$$
\bar{r}(\mathcal{E})= \begin{cases}0 & \text { if } \mathcal{E}=\boldsymbol{e}_{0} \\ 0.03 & \text { if } \mathcal{E}=\boldsymbol{e}_{1} \\ 0.06 & \text { if } \mathcal{E}=\boldsymbol{e}_{2}\end{cases}
$$

Letting $\Gamma_{k}=1.5$ and $\Omega_{k}=[0.0,0.5] \times[0.0,0.10]$, the ultraparabolic Hamilton-Jacobi system of Equations (4.1) for the value function $v\left(t, b, r, p_{\text {def }}\right)=\left(v_{0}, v_{1}, v_{2}\right)$ associated with the ultradiffusion (5.1) is then

$$
v_{0}\left(t, b, r, p_{\text {def }}\right)=0
$$

for all $\left(t, b, r, p_{\text {def }}\right) \in[0, T] \times\left[0, \Gamma_{k}\right] \times \bar{\Omega}_{k}$,

$$
\begin{aligned}
& \frac{\partial v_{1}}{\partial t}+r b \frac{\partial v_{1}}{\partial b}+\frac{1}{2} r \sigma_{\varepsilon}^{2} \frac{\partial^{2} v_{1}}{\partial r^{2}}+\alpha_{\varepsilon}\left(\bar{r}_{\varepsilon}-r\right) \frac{\partial v_{1}}{\partial r}+\frac{1}{2} p_{\text {def }}\left(0.1-p_{\text {def }}\right)(0.05)^{2} \frac{\partial^{2} v_{1}}{\partial p_{d e f}^{2}} \\
& +0.01\left(0.05-p_{\text {def }}\right) \frac{\partial v_{1}}{\partial p_{\text {def }}}-0.02 v_{1}+(0.95-1) v_{1}+0.05 v_{2}=0
\end{aligned}
$$

for all $\left(t, b, r, p_{\text {def }}\right) \in(0, T) \times\left(0, \Gamma_{k}\right) \times \Omega_{k}$, such that

$$
v_{1}\left(t, b, r, p_{\text {def }}\right)=\max \{b-0.70,0\},
$$

for $\left(t, b, r, p_{\text {def }}\right) \in(0, T) \times\left(0, \Gamma_{k}\right) \times \partial \Omega_{k}$ and

$$
\begin{aligned}
& v_{1}\left(T, b, r, p_{\text {def }}\right)=\max \{b-0.70,0\},\left(b, r, p_{\text {def }}\right) \in\left(0, \Gamma_{k}\right) \times \Omega_{k} \\
& v_{1}\left(t, \Gamma_{k}, r, p_{\text {def }}\right)=\max \{b-0.70,0\},\left(t, r, p_{\text {def }}\right) \in(0, T) \times \Omega_{k}
\end{aligned}
$$

and

$$
\begin{aligned}
& \frac{\partial v_{2}}{\partial t}+r b \frac{\partial v_{2}}{\partial b}+\frac{1}{2} r \sigma_{\varepsilon}^{2} \frac{\partial^{2} v_{2}}{\partial r^{2}}+\alpha_{\varepsilon}\left(\bar{r}_{\varepsilon}-r\right) \frac{\partial v_{2}}{\partial r}+\frac{1}{2} p_{\text {def }}\left(0.1-p_{\text {def }}\right)(0.05)^{2} \frac{\partial^{2} v_{2}}{\partial p_{\text {def }}^{2}} \\
& +0.01\left(0.05-p_{\text {def }}\right) \frac{\partial v_{2}}{\partial p_{\text {def }}}-0.02 v_{1}+\left(0.10-p_{\text {def }}\right) v_{1}+(0.9-1) v_{2}=0
\end{aligned}
$$

for all $\left(t, b, r, p_{\text {def }}\right) \in(0, T) \times\left(0, \Gamma_{k}\right) \times \Omega_{k}$, such that

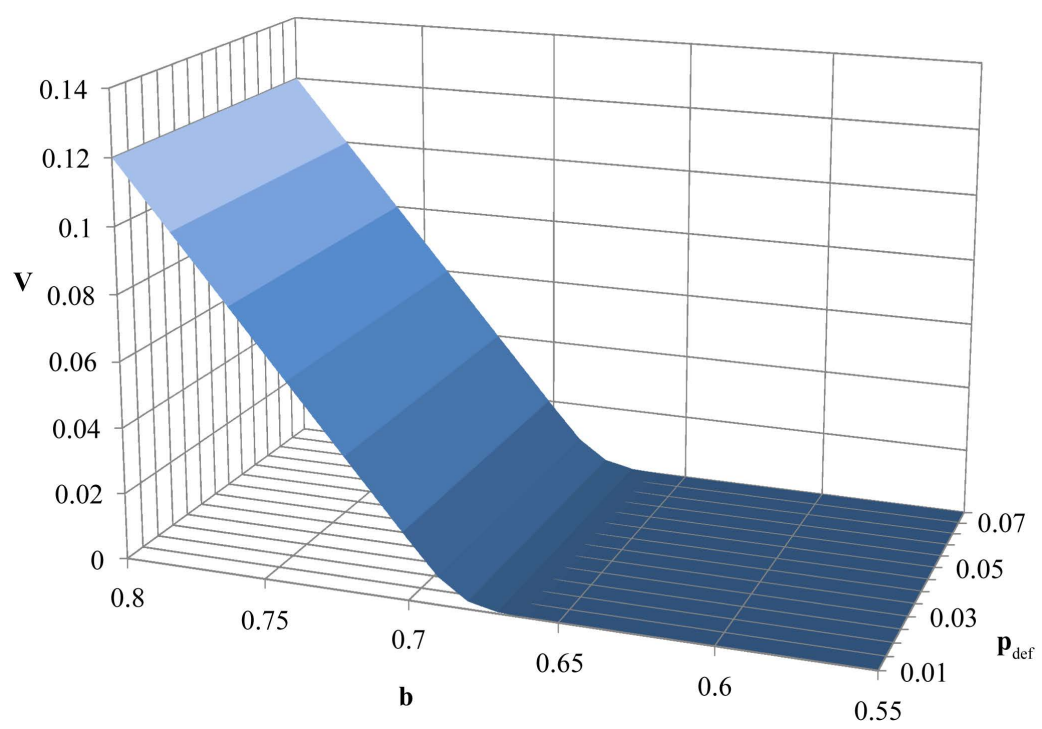

Figure 1. $v_{1}\left(0, b, 0.05, p_{d e f}\right)$. 


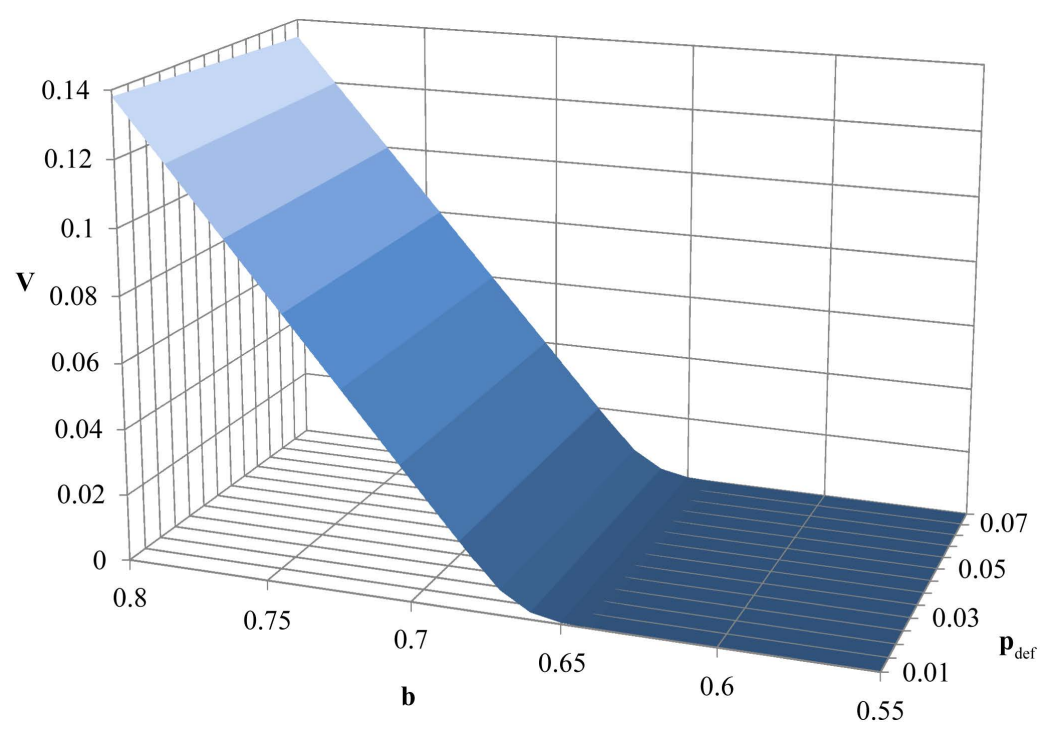

Figure 2. $v_{2}\left(0, b, 0.05, p_{d e f}\right)$.

$$
v_{2}\left(t, b, r, p_{d e f}\right)=\max \{b-0.68,0\}
$$

for $\left(t, b, r, p_{\text {def }}\right) \in(0, T) \times\left(0, \Gamma_{k}\right) \times \partial \Omega_{k}$ and

$$
\begin{aligned}
& v_{2}\left(T, b, r, p_{\text {def }}\right)=\max \{b-0.68,0\},\left(b, r, p_{\text {def }}\right) \in\left(0, \Gamma_{k}\right) \times \Omega_{k} \\
& v_{2}\left(t, \Gamma_{k}, r, p_{\text {def }}\right)=\max \{b-0.68,0\},\left(t, r, p_{\text {def }}\right) \in(0, T) \times \Omega_{k} .
\end{aligned}
$$

Figure 1 and Figure 2 show the value function components $v_{1}\left(t, b, r, p_{d e f}\right)$ and $v_{2}\left(t, b, r, p_{d e f}\right)$, respectively, for $r=0.05$. Relative to the discretization of (5.2)-(5.4), we utilized $\delta_{\tau}=0.001, \delta_{\varsigma}=0.001, \delta_{r}=0.005$, $\delta_{p}=0.005$. In particular, we note the effect of the rating based exercise prices on $v_{1}$ and $v_{2}$ and the decreasing value of $v_{2}$ with increasing $p_{\text {def }}$, as expected.

\section{References}

[1] Bielicki, T. and Rutkowski, M. (2002) Credit Risk: Modeling, Valuation and Hedging. Springer-Verlag, Berlin.

[2] Lando, D. (1998) On Cox Processes and Credit Risky Securities. Review of Derivative Research, 2, 99-120. http://dx.doi.org/10.1007/BF01531332

[3] Duffie, D. and Singleton, K. (1999) Modeling Term Structures of Defaultable Bonds. Review of Financial Studies, 12, 687-720. http://dx.doi.org/10.1007/BF01531332

[4] Longstaff, F. and Schwartz, E. (1995) Valuing Credit Derivatives. The Journal of Fixed Income, 5, 6-12. http://dx.doi.org/10.3905/jfi.1995.408138

[5] Jobst, N. and Zenios, S.A. (2005) On the Simulation of Interest Rate and Credit Risk Sensitive Securities. European Journal of Operational Research, 161, 298-324. http://dx.doi.org/10.1016/j.ejor.2003.08.044

[6] Jarrow, R., Lando, D. and Turnbull, S. (1997) A Markov Model for the Term Structure of Credit Risk Spreads. Review of Financial Studies, 10, 481-523. http://dx.doi.org/10.1093/rfs/10.2.481

[7] Das, S. and Tufano, P. (1996) Pricing Credit Sensitive Debt When Interest Rates, Credit Ratings and Credit Spreads Are Stochastic. Journal of Financial Engineering, 5, 161-198.

[8] Arvantis, A., Gregory, J. and Laurent, J.-P. (1999) Building Models for Credit Spreads. The Journal of Derivatives, 6, 27-43.

[9] Elliott, R.J. and Mamon R.S. (2002) An Interest Rate Model with a Markovian Mean Reverting Level. Quantitative Finance, 2, 454-458. http://dx.doi.org/10.1080/14697688.2002.0000012

[10] Wu, S. and Zeng, Y. (2005) A General Equilibrium Model of the Term Structure of Interest Rates under RegimeSwitching Risk. International Journal of Theoretical and Applied Finance, 8, 1-31.

[11] Harrison, J.M. and Pliska, S. (1981) Martingales and Stochastic Integrals in the Theory of Continuous Trading. Sto- 
chastic Processes and Their Applications, 11, 215-260. http://dx.doi.org/10.1016/0304-4149(81)90026-0

[12] Jarrow, R. and Turnbull, S. (1995) Pricing Derivatives on Financial Securities Subject to Credit Risk. Journal of Finance, 50, 53-85. http://dx.doi.org/10.1111/j.1540-6261.1995.tb05167.x

[13] Karlin, S. and Taylor, H. (1975) A First Course in Stochastic Processes. Academic Press, New York.

[14] Elliott, R.J., Aggoun, L. and Moore, J.B. (1994) Hidden Markov Models: Estimation and Control. Springer-Verlag, New York.

[15] Brémaud, P. (1998) Markov Chains: Gibbs Fields, Monte Carlo Simulation, and Queues. Springer-Verlag, New York.

[16] Marcozzi, M.D. (2001) On the Approximation of Optimal Stopping Problems with Application to Financial Mathematics. SIAM Journal on Scientific Computing, 22, 1865-1884. http://dx.doi.org/10.1137/S1064827599364647

[17] Marcozzi, M.D. (2015) Optimal Control of Ultradiffusion Processes with Application to Mathematical Finance. International Journal of Computer Mathematics, 92, 296-318. http://dx.doi.org/10.1080/00207160.2014.890714 
Scientific Research Publishing (SCIRP) is one of the largest Open Access journal publishers. It is currently publishing more than 200 open access, online, peer-reviewed journals covering a wide range of academic disciplines. SCIRP serves the worldwide academic communities and contributes to the progress and application of science with its publication.

Other selected journals from SCIRP are listed as below. Submit your manuscript to us via either submit@scirp.org or Online Submission Portal.
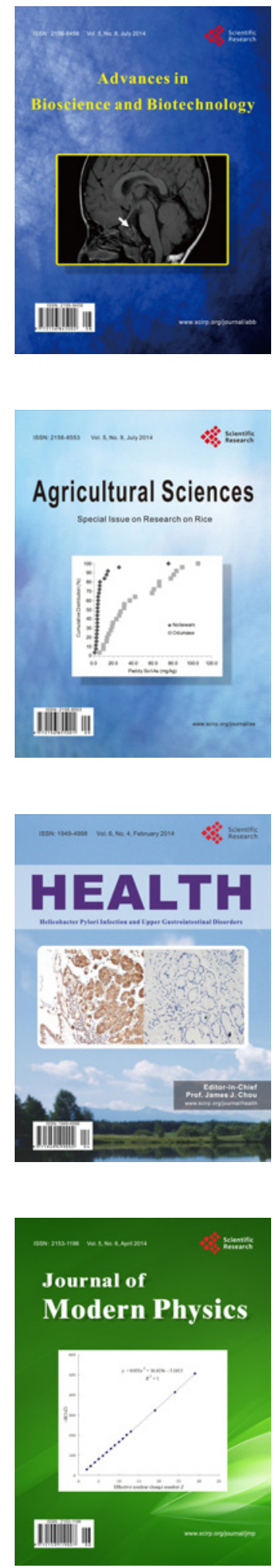
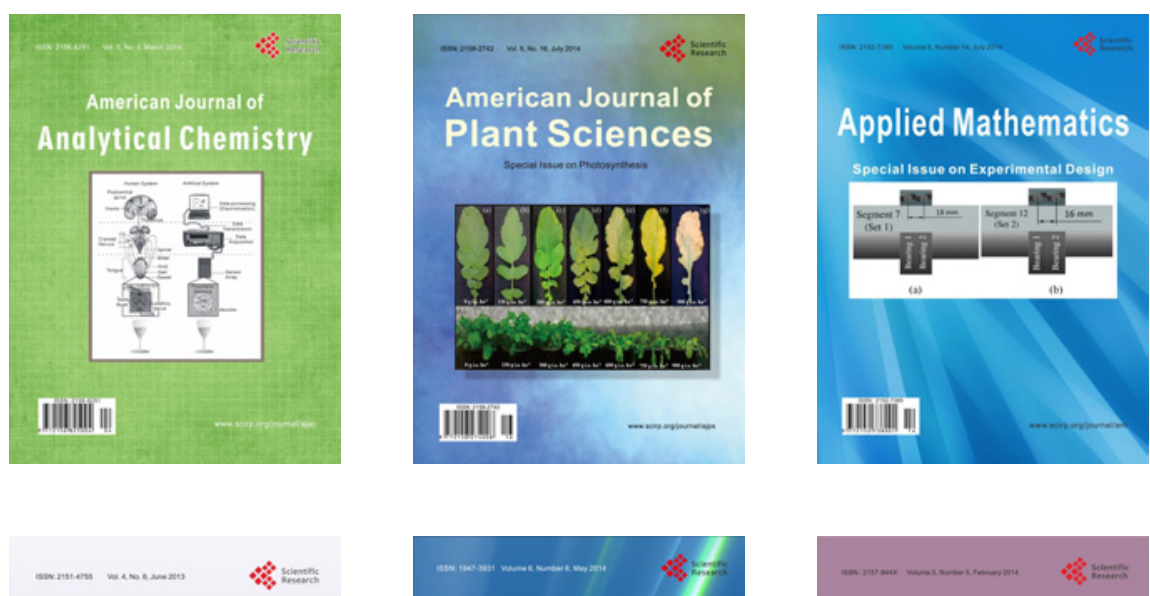

Creative Education
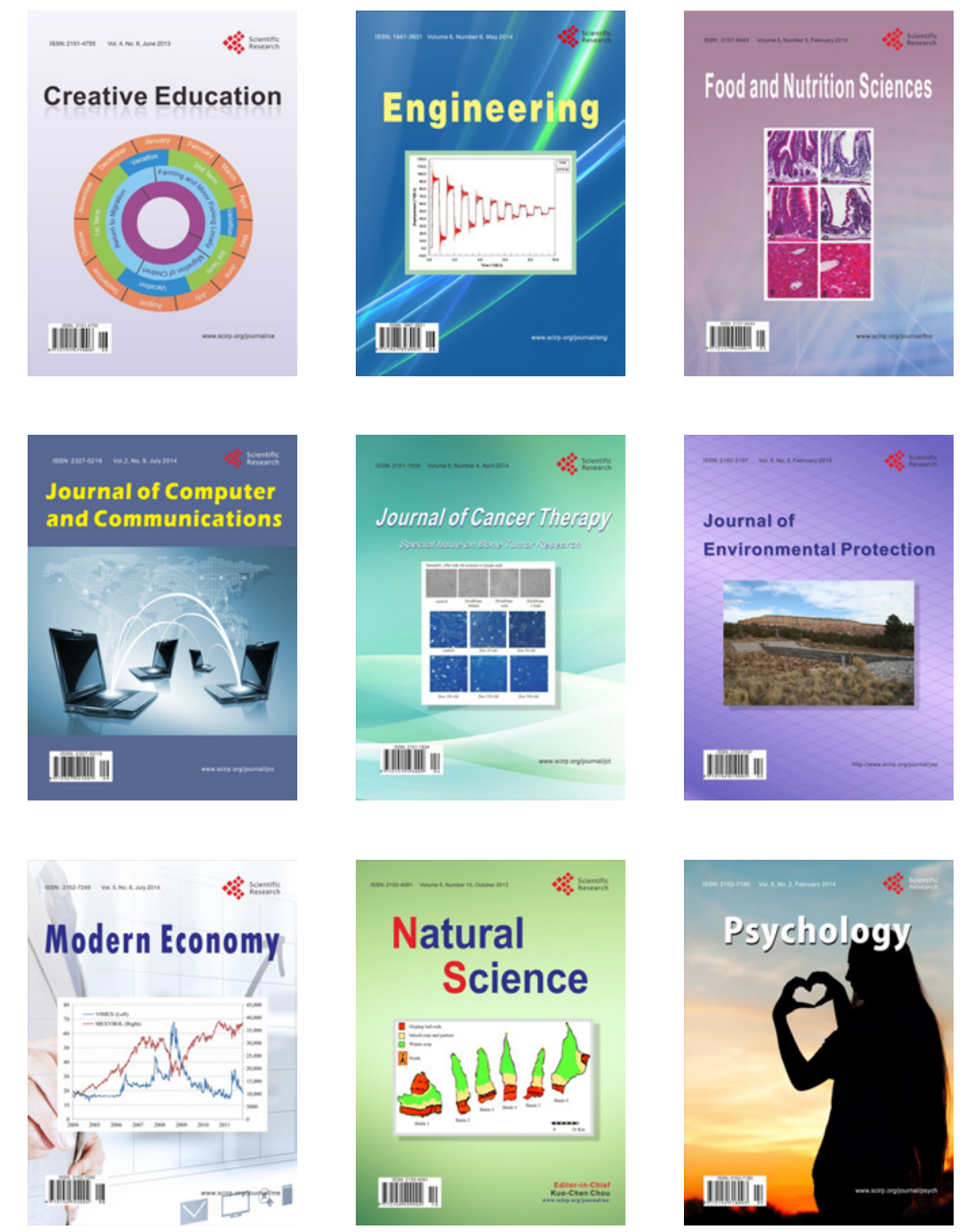